\title{
Photonic band structure and emission characteristics of a metal-backed polymeric distributed feedback laser
}

\author{
Piers Andrew ${ }^{\mathrm{a})}$ \\ Thin Film Photonics Group, School of Physics, University of Exeter, Stocker Road, Exeter, \\ Devon, EX4 4QL United Kingdom \\ Graham A. Turnbull and Ifor D. W. Samuel \\ Ultrafast Photonics Collaboration, School of Physics and Astronomy, University of St Andrews, \\ North Haugh, St Andrews, Fife, KY16 9SS United Kingdom \\ William L. Barnes \\ Thin Film Photonics Group, School of Physics, University of Exeter, Stocker Road, Exeter, \\ Devon, EX4 4QL United Kingdom
}

(Received 13 March 2002; accepted for publication 30 May 2002)

Optical losses associated with the metallic contacts necessary for charge injection are an obstacle to the development of an electrically pumped polymer laser. We show that it may be possible to overcome these losses by demonstrating the operation of a distributed-feedback polymer laser fabricated upon a silver substrate. The device lasing threshold was $\sim 150$ times greater than that of an otherwise similar metal-free device, though similar to early polymer lasers. The device emission characteristics correlated well with the measured photonic band structure, allowing an explanation of the effect of the microstructure on device operation. (c) 2002 American Institute of Physics.

[DOI: $10.1063 / 1.1496497$ ]

Semiconducting conjugated polymers are attractive gain media for optoelectronic devices due to their broad spectral emission range, good luminescence qualities, and ease of processing from solution. These properties suggest that it should be possible to construct compact electrically pumped lasers using polymer materials if the lasing threshold can be made sufficiently low.

To date, however, electrically pumped operation of an organic laser has only been demonstrated for a molecular crystal using field-effect electrodes; ${ }^{1}$ all polymer-based lasers have so far been optically pumped. One reason for this is the need to include a metallic cathode in close proximity to the gain medium to inject electrons. This is a problem because the large absorption loss of metals at optical wavelengths inhibits lasing. In the drive towards developing an electrically driven polymeric laser, it is important to demonstrate that an optically pumped laser can operate in the presence of a metal. Previously, this has been achieved for hybrid distributed Bragg reflector/metal microcavities ${ }^{2}$ and microrings. ${ }^{3}$ Perhaps the most promising design is, however, the distributed feedback (DFB) laser. ${ }^{4}$ These are easily fabricated on a large scale using embossing techniques, ${ }^{5}$ possess a high $Q$ and, hence, exhibit some of the lowest lasing thresholds observed. ${ }^{6}$ By corrugating the substrate in two directions, two-dimensional DFB lasing is possible, giving reduced thresholds and increased slope efficiencies. ${ }^{7}$

In this work we have fabricated a polymer DFB laser directly upon a metallic substrate, demonstrating that lasing is possible in the presence of a metal electrode in this technologically important geometry. Furthermore, by measuring the photonic band structure of the device we are able to relate the spectral and spatial dependence of the photolumi-

${ }^{a)}$ Electronic mail: pandrew@exeter.ac.uk nescence (PL) and lasing emission to the available photonic modes of the system. ${ }^{8}$

The DFB laser consists of a thin layer of the polymer poly[2-methoxy-5-(2'-ethylhexyloxy)-1,4-phenylene vinylene] (MEH-PPV) deposited onto a silver grating substrate to form a corrugated, asymmetric waveguide [Fig. 1(a)]. The thickness of the MEH-PPV guiding layer was such that, within the gain region of the MEH-PPV emission spectrum, only the first transverse electric waveguide mode $\left(\mathrm{TE}_{0}\right)$ was supported.

Standard holographic techniques were used to write a grating profile into a photoresist film spun on a planar silica substrate. The profile was transfered into the silica by reactive ion etching and an optically thick silver film $(\sim 127 \mathrm{~nm})$ evaporated onto the substrate. The guiding layer was fabricated by spin coating a thin film $(\sim 135 \mathrm{~nm})$ of MEH-PPV onto the silver grating from a chlorobenzene solution. The structure was characterized using noncontact atomic force microscopy (AFM) imaging after concluding the optical measurements. Figure 1(b) displays AFM images of the silver substrate and MEH-PPV top surface, showing that the deposition of a polymer layer has considerably planarized

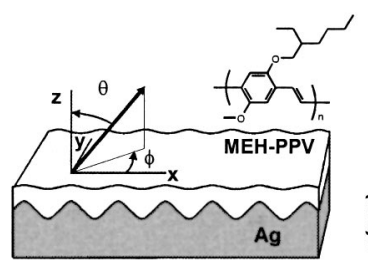

(a)

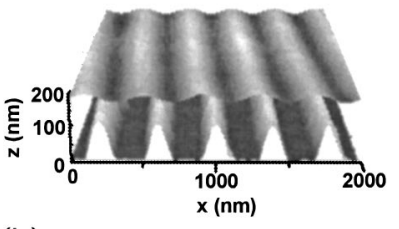

(b)
FIG. 1. (a) Schematic of the DFB laser structure, together with the MEH-PPV polymer repeat unit. (b) Atomic force microscope images of the silver grating and, offset, the MEH-PPV polymer surface. 


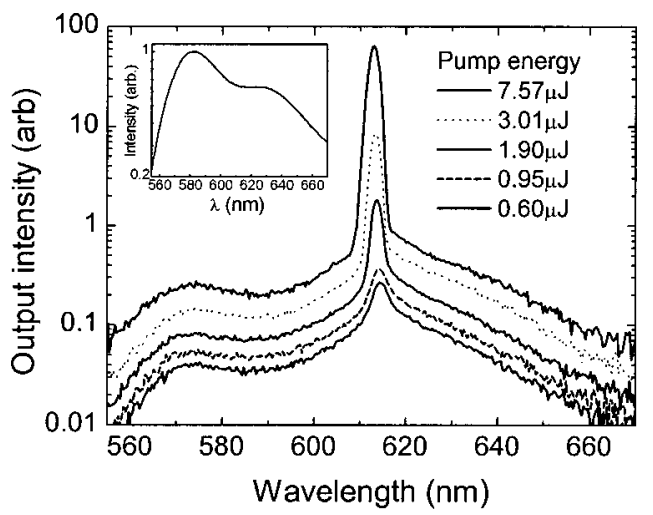

FIG. 2. TE-polarized emission spectra of the DFB laser (normalized to the peak intensity at the lasing threshold) for pump energies above and below the lasing threshold of $1.5 \mu \mathrm{J}$; the inset shows the emission spectrum of a planar film of MEH-PPV.

the grating surface. Fourier components $a_{0} \cos k_{g} x$ $+a_{1} \cos 2 k_{g} x$, where $k_{g}=2 \pi / \lambda_{g}$ is the grating Bragg vector, were fitted to the profile data to recover the harmonic content of the gratings. For the silver substrate we obtained $\lambda_{g}$ $=400 \pm 5 \mathrm{~nm}, a_{0}=52 \pm 1 \mathrm{~nm}$ and $a_{1}=20 \pm 2 \mathrm{~nm}$, while for the MEH-PPV surface, $a_{0}=10 \pm 0.1 \mathrm{~nm}$ and $a_{1}=0.5$ $\pm 0.1 \mathrm{~nm}$.

Laser emission from the DFB device was excited using $\sim 500$ ps pulses from a dye laser $(\lambda=500 \mathrm{~nm}, 10 \mathrm{~Hz})$. Pump light was incident upon the device at $45^{\circ}$ and focused to a $140 \mu \mathrm{m}$-diameter spot. TE-polarized emission was collected normal to the sample using a fiber-coupled spectrometer, giving a spectral resolution of $2.5 \mathrm{~nm}$ and angular resolution of $\sim 1^{\circ}$. The emission spectrum of the device was measured as a function of incident power while the device was maintained in a vacuum of $10^{-4}$ mbar.

Figure 2 shows the spectral dependence of the $\theta=\phi$ $=0^{\circ}$ emission for pump energies ranging from below to well above the lasing threshold of $\sim 1.5 \mu \mathrm{J}$. The spectra for the lowest excitation energies scale in intensity and resemble the MEH-PPV emission spectrum for a planar sample (see inset) with an additional superposed feature between 600 and $640 \mathrm{~nm}$. This feature is sharply peaked at $\sim 614 \mathrm{~nm}$, and is due to first-order Bragg scattered radiation from the $\mathrm{TE}_{0}$ waveguide mode. As the pump energy increases, the peak narrows and increases in intensity eventually dominating the spectra. The pump pulse energy dependence of the intensity and spectral width of this peak are given in Fig. 3, showing a transition in device behavior indicative of lasing above a threshold of $1.5 \mu \mathrm{J}$. This value is comparable with other organic lasers that include a metal contact, ${ }^{2,3}$ though is significantly higher than the threshold of $0.01 \mu \mathrm{J}$ that we measure for a metal-free DFB laser constructed on the same substrate and operating at the same wavelength. The amplified spontaneous emission peak in a MEH-PPV film of the thickness used here occurs at $\sim 620 \mathrm{~nm}$, where the gain is maximum, narrowing to a full width half maximum of $\sim 10 \mathrm{~nm}$ with increasing pump energy. ${ }^{9}$ This is significantly broader than the lasing peak observed at $614 \mathrm{~nm}$, which is coincident with the $\mathrm{TE}_{0}$ mode band edge of the structure (Fig. 4). The standing waves associated with this band edge provide the feedback necessary for lasing. Although the measured spectral mode width above threshold is limited by the resolution

Downloaded 18 Mar 2005 to 144.173.6.75. Redistribution subject

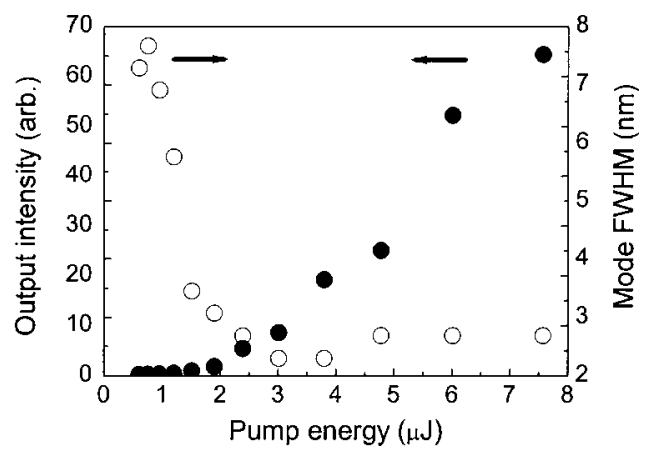

FIG. 3. Intensity of the lasing peak normalized to the threshold value (solid circles) and spectral mode full width half maximum (hollow circles) as a function of the pump energy.

of the spectrometer, the slight broadening observed in Fig. 3 could be due to other axial modes coming above threshold at higher pump energies. ${ }^{7}$

The photonic band structure of the device was determined by recording the wavelength and angle dependence of both the sample PL and reflectivity. Angle-resolved cw PL (below threshold) was excited using light from a HeNe laser $(\lambda=543.5 \mathrm{~nm},<0.5 \mathrm{~mW}, \sim 100 \mu \mathrm{m}$ diameter spot $)$. TEpolarized emission was detected with $\sim 0.2^{\circ}$ angular resolution using a spectrometer (resolution $\sim 1 \mathrm{~nm}$ ).

The technique and apparatus used to measure the reflectivity have been described elsewhere. ${ }^{10}$ Coupling of incident light to bound modes of the device was observed as a reflectivity dip, the magnitude of which corresponded to the coupling strength. The angle and wavelength dependence of the sample reflectivity was recorded to determine the waveguide mode dispersion with the in-plane wave vector and energy of the probe light, revealing the photonic band structure of the device. The spectral and angular resolutions of the apparatus were $1.5 \mathrm{~nm}$ and $0.05^{\circ}$, respectively.

The TE-polarized, $\phi=0^{\circ}$ energy (wavelength) and wave vector (emission angle) dependent PL and reflectivity of the device are given in Figs. 4(a) and 4(b). The two graphs strongly resemble one another; each is dominated by the dispersion of the $\mathrm{TE}_{0}$ waveguide mode, having two discrete branches that anticross at normal incidence. For PL these branches are emission peaks corresponding to PL emitted into the $\mathrm{TE}_{0}$ waveguide mode of the MEH-PPV layer and subsequently Bragg scattered out of the guide by the fundamental component, $k_{g}$, of the grating profile. For reflectivity, the branches are reflection minima, caused by incident light

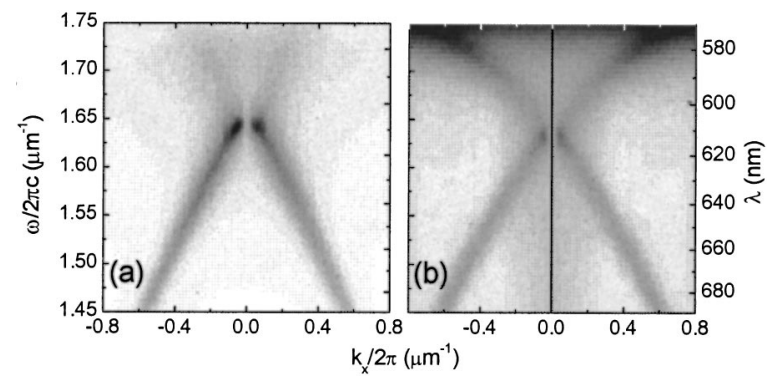

FIG. 4. PL below threshold (a) and reflectivity (b) of the laser as a function of both energy and wave vector of TE-polarized light. Dark corresponds to strong emission (a), and low reflectivity (b), respectively. The line for $k_{x}$

$\sim 0$ in (b) indicates missing data, as reflectivity could not be measured for small wave vectors (angles). 
being coupled into the $\mathrm{TE}_{0}$ guided mode by $k_{g}$ grating scatter.

The lower branch PL intensity increases near $k_{x}=0$, as the $\mathrm{TE}_{0}$ mode energy approaches the MEH-PPV peak emission, reaching a maximum at $\sim 610 \mathrm{~nm}$ (near $k_{x}=0$ ) as the branches flatten into band edges. As a result of this flattening, the mode density per unit energy is a maximum at the band edges, providing strong coupling between the MEHPPV PL and the $\mathrm{TE}_{0}$ mode, and hence, strong reradiation through Bragg scattering. Maximum emission is expected at the band edge, however, there is a gap between the positive and negative $k_{x}$ branches at $k_{x}=0$ and thus a minimum in Bragg scattered reradiation. It is at this band edge, between the two PL peaks at $\lambda \sim 610 \mathrm{~nm}, \theta= \pm 2.5^{\circ}(\omega / 2 \pi c$ $\left.=1.64 \mu \mathrm{m}^{-1}, k_{x} / 2 \pi= \pm 0.07 \mu \mathrm{m}^{-1}\right)$ that the device lases. This behavior is also seen in the reflectivity, with maximum coupling occurring for the lower energy branch at $\lambda$ $=614 \mathrm{~nm}, \quad \theta= \pm 2.5^{\circ} \quad\left(\omega / 2 \pi c=1.63 \mu \mathrm{m}^{-1}, k_{x} / 2 \pi\right.$ $\left.= \pm 0.07 \mu \mathrm{m}^{-1}\right)$, almost coincident with the PL maxima. Between these reflectivity minima, the reflectivity is high, indicating minimal coupling of incident light to the guided modes, agreeing with the gap in the PL emission branches.

The strong correlation between device PL and reflectivity is due to the influence of first order Bragg scattering on both processes. Similar correlation has been observed between the emission from, and the transmission through, a DFB laser fabricated on a silica substrate. ${ }^{8}$ In both cases the device emission is strongly influenced by the photonic band structure, so probing this structure by reflectivity and/or transmission measurements is a powerful aid in the design and understanding of polymer lasers.

In Fig. 4, the two branches arise from Bragg scattered $\mathrm{TE}_{0}$ waveguide modes originally propagating in the positive and negative $x$ directions. The angular position, $\theta$ of maximum coupling between incident light and the mode is determined by the conservation of in-plane momentum, including the addition or subtraction of an integer number, $m$ of Bragg vectors (to account for grating scattering), according to $k_{x}$ $=k_{0} \sin \theta= \pm k_{\text {mode }} \pm m k_{g}$. Here, $k_{x}$ is the in-plane wave vector of the light coupling to/from the mode and $k_{\text {mode }}$ is the waveguide mode wave vector. Branches with positive (negative) gradient arise from a $-k_{g}, m=-1\left(+k_{g}, m=+1\right)$ scatter of guided modes propagating in the positive (negative) $x$ direction. Coupling of light into and out of the guide thus arises from single $k_{g}$ scattering, and so the coupling strength is primarily dependent on the amplitude of the fundamental grating profile component.

The normal incidence anticrossing behavior occurs because the modes coupling at $k_{x}=0$ have momentum $\pm k_{g}$ within the guide, and can couple by $2 k_{g}$ scattering. The resulting interference between counter-propagating modes leads to two standing-wave solutions at $k_{x}=0$, each with periodicity $\lambda_{g}$, but with different field distributions. ${ }^{11}$ These standing waves form band edges, with differing energies for the same wave vector, creating a band gap for the propagation of guided modes and leading to the anticrossing observed in Figs. 4(a) and 4(b). Since this process depends on the guided modes undergoing $2 k_{g}$ scattering, gap formation is primarily dependent on the amplitude of the second har- monic component of the grating profile, with Bragg vector $2 k_{g}$. In our system, the energy gap is somewhat indistinct due to the poorly coupled upper energy band edge, and is thus dominated by the obvious wave vector gap for the lower branch at $k_{x}=0$. This "false" gap results from the absence of coupling between waveguide modes and light, rather than the absence of waveguide modes themselves.

The reflectivity data of Fig. 4(b) shows that neither band edge couples well to incident light (there are no reflectivity minima at $k_{x}=0$ ), which is a result of the field distributions of the modes with respect to the grating profile. ${ }^{11}$ The upper band edge is overcoupled, leading to a lossy low- $Q$ resonance, while the lower band edge is uncoupled. This reduced radiative coupling at the lower band edge is the key to the operation of the device as a laser, since the feedback induced by the $2 k_{g}$ scattering suffers less competition with the radiative loss of emission scattered out of the guide by single $k_{g}$ scattering. ${ }^{8}$ The differing radiation losses of the two band edges acts as a frequency selection mechanism, with the poorly coupled branch having a lower threshold than the well-coupled branch. ${ }^{8,12}$ In common with many other DFB lasers, lasing occurs at the lower energy band edge rather than the upper, although upper band edge lasing has been observed in circular DBR structures. ${ }^{13}$

We have demonstrated the band edge operation of a silver-backed distributed feedback polymer laser, showing that the close proximity of a metal to the gain medium does not preclude lasing in a simple device structure. The lasing threshold was $\sim 150$ times that of a metal-free device operating at the same wavelength and fabricated on the same substrate. Furthermore, reflectivity measurements have allowed us to explain the emission in terms of the photonic band structure of the device.

The authors gratefully acknowledge the EPSRC and SHEFC for financial support. IDWS is a Royal Society University Research Fellow.

${ }^{1}$ J. H. Schön, Ch. Kloc, A. Dodabalapur, and B. Batlogg, Science 289, 589 (2000).

${ }^{2}$ N. Tessler, G. J. Denton, and R. H. Friend, Nature (London) 382, 695 (1996).

${ }^{3}$ S. V. Frolov, A. Fujii, D. Chinn, Z. V. Vardeny, K. Yoshino, and R. V. Gregory, Appl. Phys. Lett. 72, 2811 (1998).

${ }^{4}$ M. D. McGehee, M. A. Díaz-García, F. Hide, R. Gupta, E. K. Miller, D. Moses, and A. J. Heeger, Appl. Phys. Lett. 72, 1536 (1998).

${ }^{5}$ M. Berggren, A. Dodabalapur, R. E. Slusher, A. Timko, and O. Nalamasu, Appl. Phys. Lett. 72, 410 (1997).

${ }^{6}$ T. Granlund, M. Theander, M. Berggren, M. Andersson, A. Ruzeckas, V. Sundstrom, G. Bjork, M. Granstrom, and O. Inganäs, Chem. Phys. Lett. 288, 879 (1998).

${ }^{7}$ S. Riechel, C. Kallinger, U. Lemmer, J. Feldmann, K. Gombert, V. Wittwer, and U. Scherf, Appl. Phys. Lett. 77, 2310 (2000).

${ }^{8}$ G. A. Turnbull, P. Andrew, W. L. Barnes, and I. D. W. Samuel, Phys. Rev. B 64, 125122 (2001).

${ }^{9}$ A. K. Sheridan, G. A. Turnbull, A. N. Safonov, and I. D. W. Samuel, Phys. Rev. B 62, R11929 (2000).

${ }^{10}$ M. G. Salt and W. L. Barnes, Opt. Commun. 166, 151 (1999).

${ }^{11}$ W. L. Barnes, T. W. Priest, S. C. Kitson, and J. R. Sambles, Phys. Rev. B 54, 6227 (1996)

${ }^{12}$ R. F. Kazarinov and C. H. Henry, IEEE J. Quantum Electron. 21, 144 (1985).

${ }^{13}$ N. Moll, R. F. Mahrt, C. Bauer, H. Giessen, B. Schnabel, E. B. Kley, and U. Scherf, Appl. Phys. Lett. 80, 734 (2002). 